\title{
Short course of high-dose steroids for anaphylaxis caused flare up of tuberculosis: A case report
}

\author{
Shital Patil, Anil Jadhav \\ MIMSR Medical College Latur India, Latur, Maharashtra, India
}

\section{ABSTRACT}

A25-year-old male patient received high-dose intravenous steroids for life-threatening anaphylaxis because of bee sting only for 4 days resulted in reactivation of latent tuberculosis infection (LTBI). Clinical presentation is acute form of progressive pulmonary tuberculosis with pleural effusion that can be misdiagnosed as a community-acquired pneumonia. High index of suspicion with adequate evaluation is must in all cases to have satisfactory treatment outcome. Bronchoscopy is crucial in evaluation with histopathology expertise is must while managing such cases.

Key words: high dose steroids, pulmonary tuberculosis, latent tuberculosis infection

\section{INTRODUCTION}

More than $30 \%$ of population in India is infected with tubercle bacilli, ${ }^{[1]}$ and accounts for nearly $20-30 \%$ of the global tuberculosis (TB) burden. After TB infection, active disease develops in 3-5\% of "immunecompetent" individuals within 2 years (defined as progressive primary TB, which is observed more commonly in patients with a large inoculation or immunosuppression), whereas active TB disease develops in an additional $3-5 \%$ of infected persons during the remainder of their life time. ${ }^{[2]}$

Corticosteroids, through their immunosuppressive and anti-inflammatory effects, impair antibody formation and cell-mediated immunity. Through these actions, steroids predispose patients to a variety of secondary infections including reactivation of latent TB foci and reinfection with Mycobacterium tuberculosis. ${ }^{[3,4]}$ In this case report, we observed reactivation of $\mathrm{TB}$ with the use of short course of steroids used for non-pulmonary indication.

\section{CASE REPORT}

A 25 year-old male, non-alcoholic, nontobacco addict patient presented to intensive care unit with a history of $>100$ honey bee sting with high-grade fever, shortness of breath, and convulsions for $3 \mathrm{~h}$. He had fever, hypotension, and tachycardia on evaluation. Hematologic parameters were showing leucocytosis with high titers of C-reactive protein (CRP) and normal liver and kidney functions. Chest X-ray scanning was performed, which was normal (Figure 1).

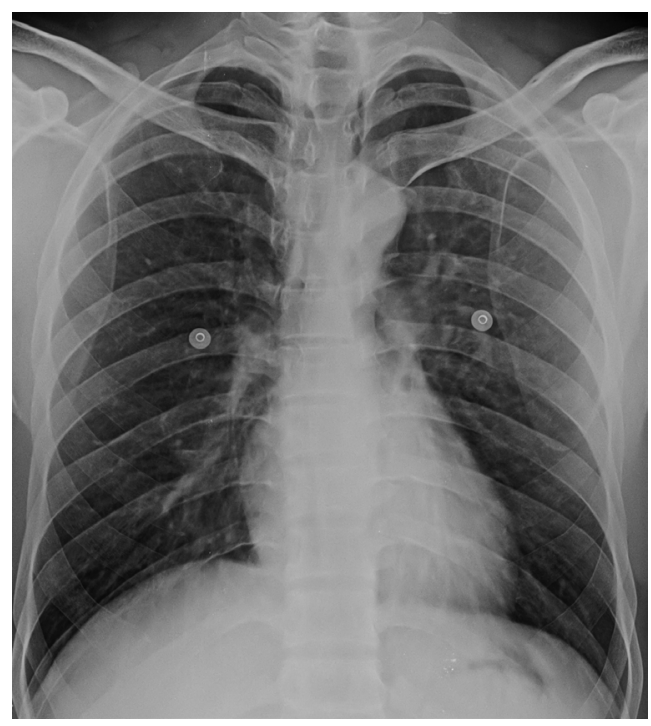

Figure 1: Normal lung parenchyma with normal hilum, and cardiophrenic and costophrenic angles are clear.

The patient received intensive care treatment with oxygen, inotropes, and high-dose intravenous methylprednisolone $125 \mathrm{mg}$ stat 
and $40 \mathrm{mg}$ three times for 3 days. The patient was discharged from hospital on the fourth day of hospitalization.

After 2 weeks of discharge from hospital, patient developed febrile respiratory illness that consists of "typical pleuritic chest pain" with minimal dry cough and shortness of breath grade II. Vital parameters were stable with normal oxygen saturation. Hemogram was normal; liver and kidney functions were normal.

The patient received empirical antibiotics, with partial response to symptoms. We repeated hematologic parameters, sputum for acid-fast bacilli evaluation, and chest X-ray after 2 weeks of high-dose intravenous steroids for 4 days (Figure 2).

After 48 hours of antibiotics therapy, repeated chest X-ray showed radiologic features of right middle lobe collapse with effusion (Figure 3). (Classical "tongue-shaped" opacity of right middle lobe collapse, crowding of right lower zone ribs, and shift of diaphragm. Horizontal line of pleural fluid just behind the diaphragm was also observed.)

Bronchoscopy showed narrowed lumen secondary to peribronchial compression or bulge (Figure 4). We preferred TBNA for diagnosis and blind transbronchial lung biopsy (TBLB).

TBNA cytology yields cellular material that consists of lymphocyte and macrophages without any evidence of granuloma, acid-fast bacilli, histiocytes, or any giant cells.

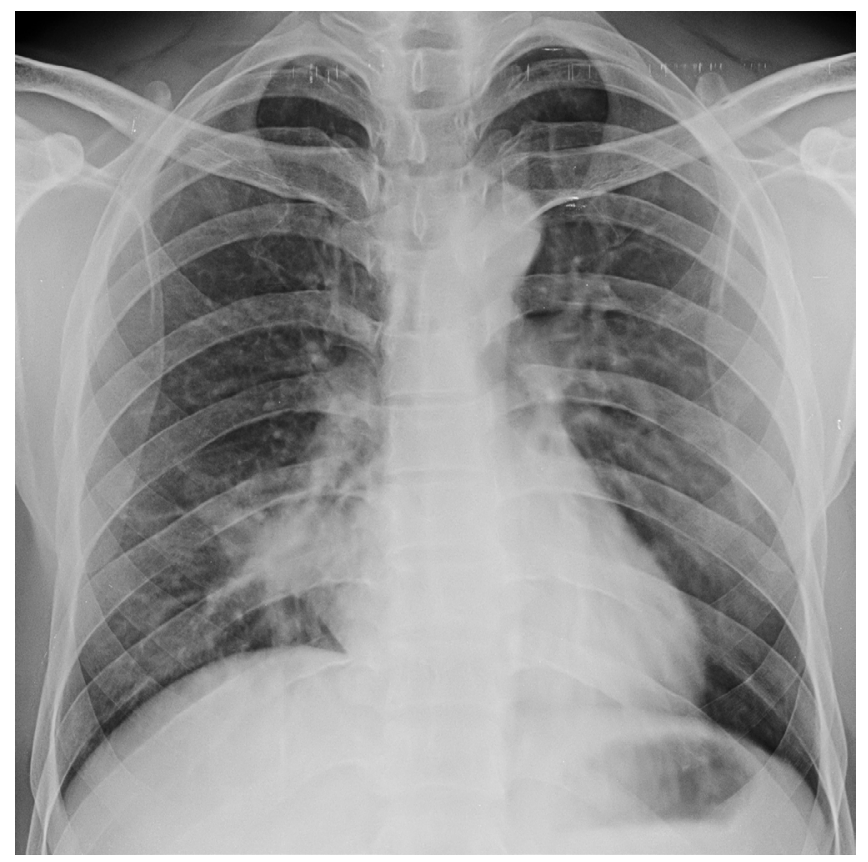

Figure 2: Right lower zone opacification/consolidation obscuring right heart border suggests consolidation of right middle lobe.
TBLB histopathology analysis showed classical epithelioid granuloma with giant cell. Bronchial wash for Gene Xpert/ MTB Rif analysis showed MTB genome detection without rifampicin mutation.

Four-drug antituberculosis treatment (ATT), consisting of isoniazid, rifampicin, ethambutol and pyrazinamide, was initiated for 2 months as intensive phase and first 3 drugs for 4 months as continuation phase as per national guidelines for treatment of TB.

After 14 days of antituberculosis therapy, chest X-ray showed radiological response to ATT, partial resolution of right middle lobe consolidation and resolving pleural effusion (Figure 5).

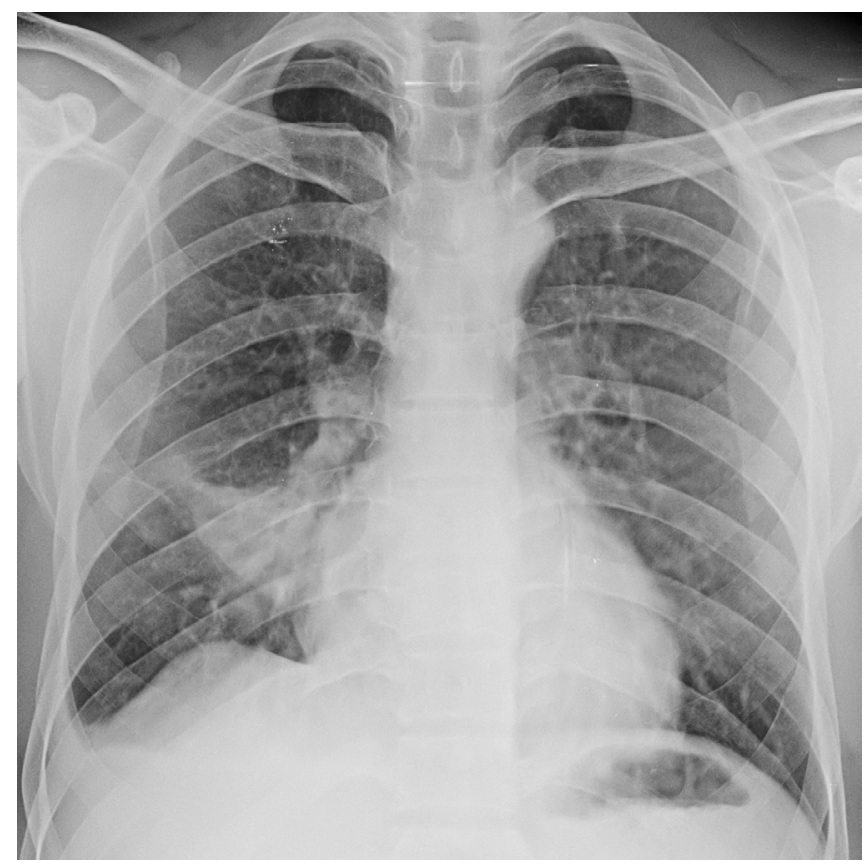

Figure 3: Radiological features of right middle lobe collapse with effusion.

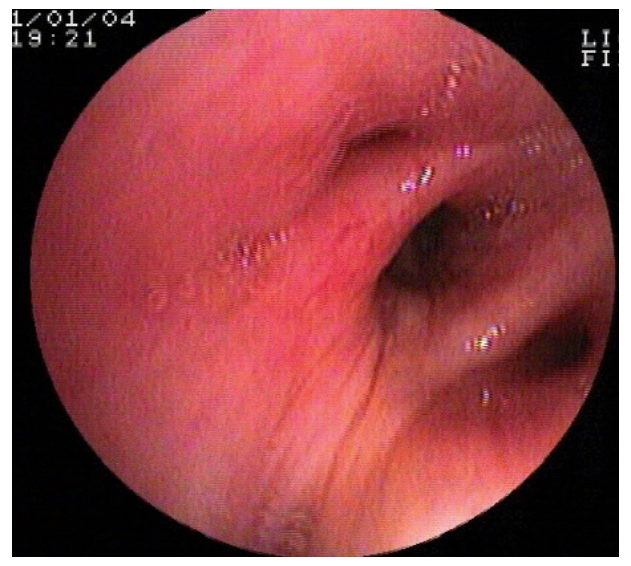

Figure 4: Bronchoscopy showed narrowed lumen secondary to peribronchial compression or bulge. 
After 6 months of ATT, repeated X-ray showed nearcomplete radiological resolution of parenchymal opacity with cardiophrenic and costophrenic angles clear (Figure 6). As compared to previous chest X-rays, after the completion of ATT, there is no obvious residual pleural thickening at costophrenic angle or any parenchymal lesion/opacity. There is "thickening of oblique fissure" with diaphragmatic tenting on right side.

\section{DISCUSSION}

Corticosteroids, through their immunosuppressive and anti-inflammatory effects, impair antibody formation and cell-mediated immunity. Through these actions, steroids predispose patients to a variety of secondary infections including reactivation of latent tuberculous foci and reinfection with $M$. tuberculosis. ${ }^{[3,4]}$

The occurrence of TB also correlates with steroid dosing. One study even suggested that for each gram of prednisolone dose, there was a $23 \%$ increment in the chance of developing TB. ${ }^{[5]}$ These effects are most evident if steroid doses exceed $0.03 \mathrm{mg} / \mathrm{kg}$ per day of prednisolone or equivalent. At doses $>1 \mathrm{mg} / \mathrm{kg}$ per day, a marked increase in susceptibility to a wide variety of infections is experienced after several weeks. Treatment for $<5$ days appears to have less effect on immune function and predisposition to infections. Continuous therapy has longer and more profound immunosuppressive effects as compared to intermittent therapy. ${ }^{[6]}$

Different systemic steroids, taken by mouth or given by injection, used in dermatology practice include hydrocortisone, prednisone, prednisolone, methylprednisolone, triamcinolone, dexamethasone, and betamethasone. These differ in their relative anti-inflammatory and mineralocorticoid activity, plasma half-life, and duration of adrenocorticotropin hormone (ACTH) suppression. Treatment regimen is called low dose if $<10-\mathrm{mg} /$ day prednisolone or equivalent is used and high dose when $>20-\mathrm{mg} /$ day prednisolone is prescribed. Similarly, steroid treatment is called short-term treatment when used for $\leq 3$ weeks and long-term when given for $>4$ weeks. ${ }^{\left[{ }^{[]}\right.}$

The role of steroid therapy in precipitating TB is controversial, while some maintain that steroid therapy might cause exacerbation of active or apparently inactive TB. ${ }^{[8]}$

\section{Steroids in tuberculosis - "double-edged sword"}

Steroids are used during the treatment of tuberculosis with cover of antituberculosis drugs to prevent exaggerated response to tuberculous proteins shown by host immune system, especially in pericardial, meningeal, and miliary TB

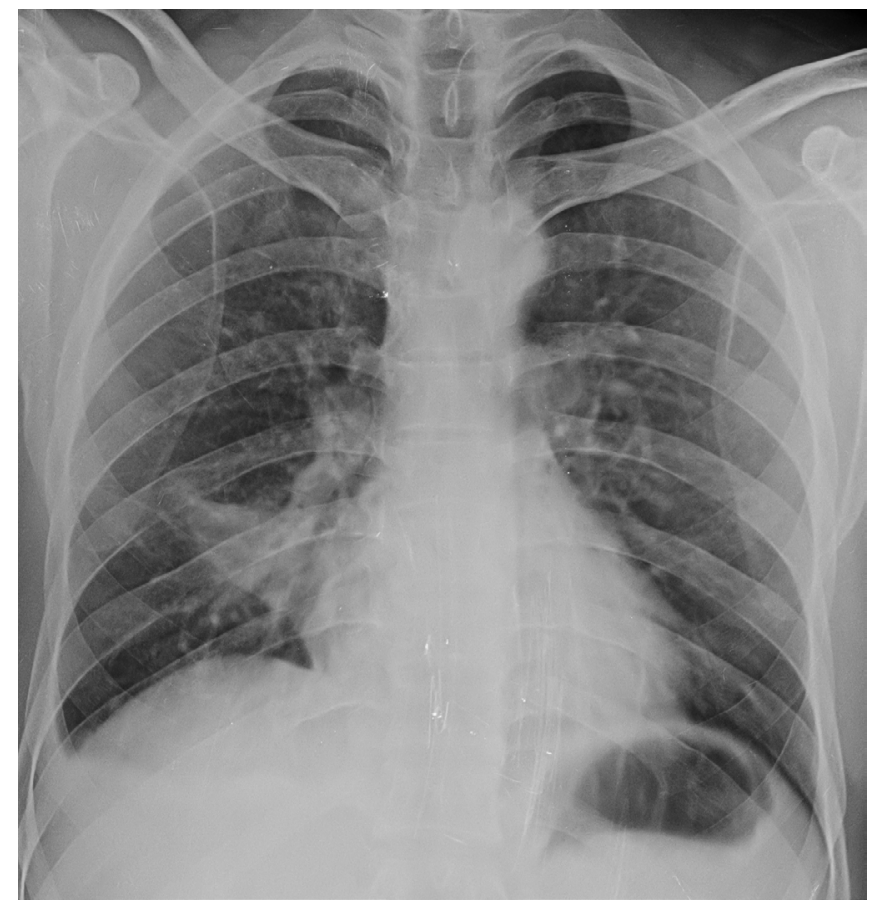

Figure 5: Radiological response to ATT, partial resolution of right middle lobe consolida

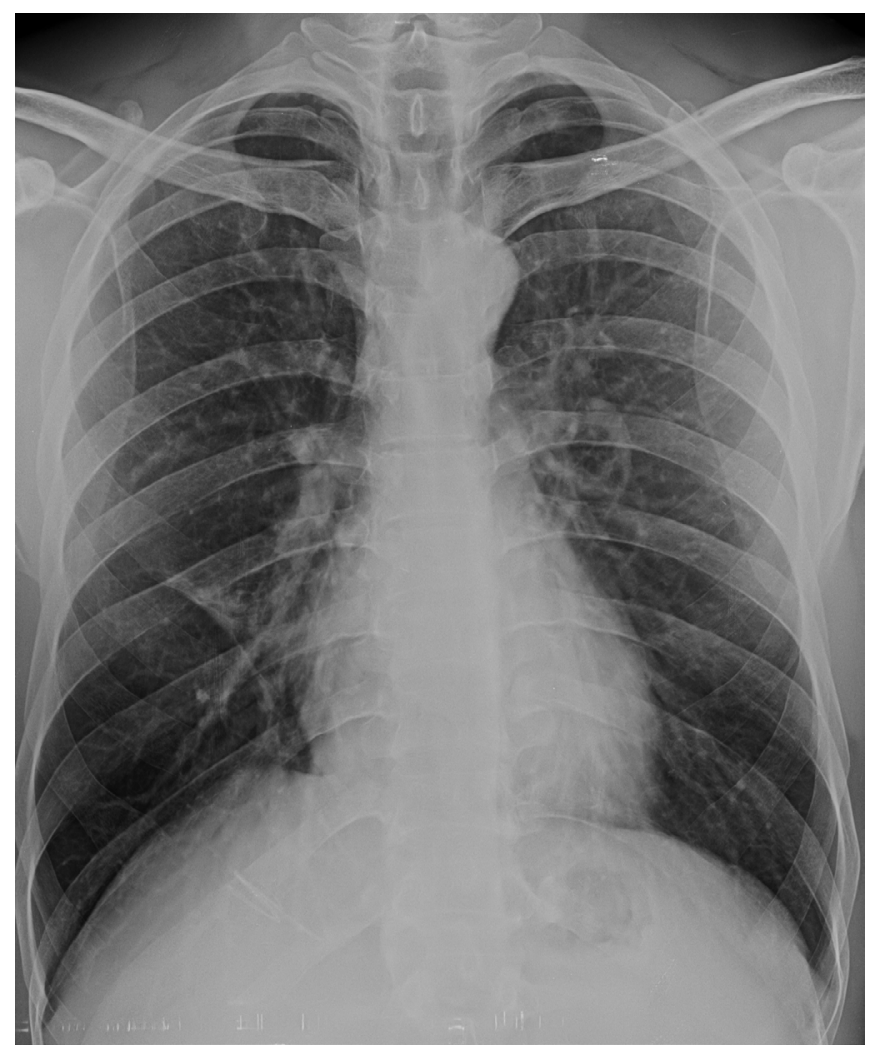

Figure 6: Near-complete radiological resolution of parenchymal opacity, with cardiophrenic and costophrenic angles clear. 
with hypoxemia and toxemia or fever persistent irrespective of treatment. Short course of steroids under the cover of ATT shown excellent response and treatment outcome by its known anti-inflammatory effect. Steroids are also used in non-pulmonary or life-threatening medical emergencies where duration and dosages are not fixed, although used for $<1$ week. Very few data regarding possible role of steroids used for short course leading to reactivation of latent tuberculosis infection (LTBI) are available.

In our case, initially patient was treated as a case of acute life-threatening anaphylaxis with high-dose intravenous steroids only for 4 days, that is, $<1$ week, resulted in complete cure of that event but resulted in flare up of latent tuberculosis infection in 2 weeks after treatment with steroid and presented as acute pulmonary tuberculosis with pleural effusion. Clinical presentation of acute pulmonary tuberculosis was confusing with community-acquired pneumonia and can be easily missed. Even right middle lobe presentation of acute pulmonary tuberculosis in less than a month is rarely described previously or very few data is available. We have evaluated with bronchoscopy, pleural fluid analysis, and histopathology of bronchoscopic biopsy specimens and confirmed to have tuberculosis is the etiology and treated with four-drug antituberculosis treatment containing isoniazid, rifampicin, pyrazinamide, and ethambutol. The response to therapy was excellent, with complete clinical and radiological response in 6 months of treatment.

\section{CONCLUSION}

Steroids will cause generalized immunosuppression and qualitative and quantitative local lung immune defense defect, resulting in increased risk of tuberculosis because of reactivation of latent infection or relapse or acquiring new infection. Risk of tuberculosis is proportional to use steroid dosage and duration either for pulmonary or nonpulmonary indication.
High index of suspicion is must to predict pulmonary tuberculosis because of confusing clinical pulmonary syndrome mimicking like community-acquired pneumonia. Always consider tuberculosis as the cause of pulmonary infiltrates, consolidation, or effusion in cases with a history of use of steroids irrespective of dosage or duration to have successful treatment outcome. Bronchoscopy is crucial in evaluation with histopathology expertise is must while managing such cases.

\section{Conflict of Interest}

None declared.

\section{REFERENCES}

1. Surpal BB. Magnitude of tuberculosis problem in India. J Ind Med Assoc1989; 87:201.

2. American Thoracic Society, Centers for Disease Control and Prevention. Targeted tuberculin testing and treatment of latent tuberculosis infection. Am J Respir Crit Care Med 2000; 161: S221-47.

3. Munck A, Mendel DB, Smith LI, Orti E. Glucocorticoid receptors and actions. Am Rev RespirDis1990; 141:82

4. Trehan AP, Ahmed A. Corticosteroids: a review with emphasis on complication of prolonged systemic therapy. Ann Allergy 1989; 62:375.

5. Tam LS, Li EK, Wong SM, Szeto CC. Risk factors and clinical features for tuberculosis among patients with systemic lupus erythematosus in Hong Kong. Scand J Rheumatol2002; 31:296-300.

6. Wilson CB. The cellular immune system and its role in host defence. In: Mandel GL, Douglas RG Jr., Bennett JE: Eds. Principles and Practice of Infectious Disease. New York: Churchill Livingstone, 1990:101.

7. MitsosL and Sasseville D. Systemic Corticosteroid Use in Dermatology: Defining, Detailing, and Demystifying. Dermatology Rounds 2011; 7:124-35.

8. Pal D, Behera D, Gupta D, Aggarwal A. Tuberculosis in patients receiving prolonged treatment with oral corticosteroids for respiratory disorders. Indian J Tub 2002; 49:83-6.

How to cite this article: Patil S, Jadhav A. Short course of high-dose steroids for anaphylaxis caused flare up of tuberculosis: A case report. J Transl Int Med 2019; 7: 39-42. 